\title{
Noncontact Modulated Laser Calorimetry for Liquid Austenitic Stainless Steel in dc Magnetic Field
}

\author{
Hiroyuki FUKUYAMA, ${ }^{11}$ Kakeru TAKAHASHI, ${ }^{11}$ Shoji SAKASHITA, ${ }^{1)}$ Hidekazu KOBATAKE, ${ }^{11}$ \\ Takao TSUKADA ${ }^{2)}$ and Satoshi AWAJ|3l
}

1) Institute of Multidisciplinary Research for Advanced Materials, Tohoku University, 2-1-1 Katahira, Aoba-ku, Sendai $980-8577$ Japan. $\quad 2$ 2) Department of Chemical Engineering, Tohoku University, 6-6-07 Aramaki, Aoba-ku, Sendai 980-8579 Japan. 3) Institute for Materials Research, Tohoku University, 2-1-1 Katahira, Aoba-ku, Sendai 980-8577 Japan.

(Received on March 31, 2009; accepted on May 7, 2009)

\begin{abstract}
A newly developed noncontact modulated laser calorimetry method was attempted to measure the isobaric specific heat capacity and thermal conductivity of liquid austenitic stainless steel (SUS 304: Japanese Industrial Standards). A stainless steel droplet was electromagnetically levitated in a radio frequency coil. A dc magnetic field of 4-5 T was superimposed to the droplet to suppress the convection in the droplet by the Lorenz force. The specific heat capacity of the liquid SUS 304 was successfully measured at temperatures ranging from 1750 to $1970 \mathrm{~K}$. No clear temperature dependence was observed. The mean value over the whole temperature range is $794 \pm 76 \mathrm{~J} \cdot \mathrm{kg}^{-1} \cdot \mathrm{K}^{-1}$.

The lower thermal conductivity of the liquid SUS 304 was obtained at higher dc magnetic field, which indicates that convection in the droplet was reduced by dc magnetic field. The thermal conductivity was measured as $60 \pm 9 \mathrm{~W} \cdot \mathrm{m}^{-1} \cdot \mathrm{K}^{-1}$ at $5 \mathrm{~T}$ in the same temperature range. For the thermal conductivity measurement, it is necessary to verify the effect of suppression of the convection by conducting the calorimetry at higher dc magnetic field.
\end{abstract}

KEY WORDS: laser calorimetry; stainless steel; dc magnetic field; heat capacity; thermal conductivity; electromagnetic levitation; welding.

\section{Introduction}

Recently, a computer-aided welding system has been developed for improving welding processes for stainless steels. ${ }^{1)}$ Thermophysical properties such as heat capacity, thermal conductivity, density and heat of fusion are required for a heat conduction model. Furthermore, surface tension is needed for a welding pool shape modeling. Accurate knowledge of thermophysical properties of stainless steel is necessary in the simulation. However, the thermophysical property data are scarce for high-temperature stainless steel melts because of the experimental difficulty arising from the contamination from the contact materials and the convections in the melt.

Fecht and co-workers ${ }^{2-5)}$ have developed the modulation calorimetry for electromagnetically levitated metallic melts to measure the heat capacity, thermal conductivity and hemispherical total emissivity. However, the thermal conductivity can not be measured because the convection still remained in the melt. Yasuda et al. ${ }^{6}$ ) have reported that an electromagnetically levitated droplet behaves as a hard body in a dc magnetic field. The oscillation and convections of the droplet were effectively suppressed by the Lorenz force in a dc magnetic field. Based on the studies described above $^{2-6)}$ the present authors have newly developed a noncontact modulated laser calorimetry for metallic melts in a dc magnetic field..$^{7-9)}$ An electromagnetic levitator is equipped in a superconducting magnet to hold a droplet statically. The high-temperature droplet is heated sinusoidally by laser irradiation on the top; then, the temperature response is measured at the bottom of the droplet to determine the heat capacity, thermal conductivity and emissivity of the droplet. Tsukada et al. ${ }^{10)}$ described the theory and validity of this technique using numerical simulation. In addition, the experimental principle was verified using a solid platinum sphere as a reference. ${ }^{7)}$ Moreover, we successfully measured the heat capacity, thermal conductivity and hemispherical total emissivity of liquid $\mathrm{Si}^{8}{ }^{89}$ Here, we attempted to apply this method for measuring the heat capacity and thermal conductivity of liquid austenitic stainless steel (SUS 304: Japanese Industrial Standards).

\section{Principle of Noncontact Modulated Laser Calo- rimetry}

\subsection{Heat Capacity}

The experimental principle is similar to that explained by the present authors. ${ }^{7-10)}$ Here, a brief outline is described. Figure 1 presents a heat flow model of noncontact modulated laser calorimetry in a gas stream. The top surface of the droplet levitated in a radio frequency ( $\mathrm{rf}$ ) coil is heated with angular frequency $\omega\left(\mathrm{rad} \cdot \mathrm{s}^{-1}\right)$ using a modulated laser 


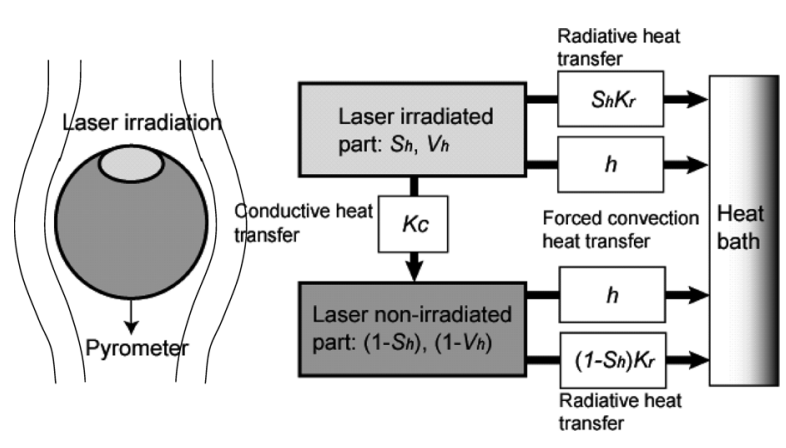

Fig. 1. Heat flow model for noncontact modulated laser calorimetry in a gas stream.

with a form of $p_{o}(1+\cos \omega t)\left(\mathrm{W} \cdot \mathrm{m}^{-2}\right)$. The temperature response at the bottom surface of the droplet is measured using a pyrometer. The heat balances in this system are as follows.

The heat balance at laser-irradiated part is

$$
\begin{array}{r}
V_{h} C_{p} \frac{d T_{h}}{d t}=Q_{h}+\alpha S_{h} A p_{o}(1+\cos \omega t)-S_{h} A \varepsilon \sigma\left(T_{h}^{4}-T_{\infty}^{4}\right) \\
-S_{h} A h\left(T_{h}-T_{\infty}\right)-K_{c}\left(T_{h}-T_{l}\right) \ldots \ldots(1)
\end{array}
$$

that at the laser-nonirradiated part is

$$
\begin{aligned}
\left(1-V_{h}\right) C_{p} \frac{d T_{l}}{d t}= & Q_{l}-\left(1-S_{h}\right) A \varepsilon \sigma\left(T_{l}^{4}-T_{\infty}^{4}\right) \\
& -\left(1-S_{h}\right) A h\left(T_{l}-T_{\infty}\right)+K_{c}\left(T_{h}-T_{l}\right)
\end{aligned}
$$

In those equations, $S_{h}$ is the fraction of the irradiated surface area that is heated by the laser, $V_{h}$ denotes the volume fraction corresponding to $S_{h}, C_{p}\left(\mathrm{~J} \cdot \mathrm{K}^{-1}\right)$ represents the isobaric heat capacity, $T(\mathrm{~K})$ is the absolute temperature, $Q$ (W) is the power input from the rf coil, $\alpha$ is the absorptivity, $A\left(\mathrm{~m}^{2}\right)$ is the surface area of the droplet, $h(\mathrm{~W}$. $\left.\mathrm{m}^{-2} \cdot \mathrm{K}^{-1}\right)$ is the forced convection heat transfer coefficient of a gas surrounding the sample droplet, $K_{c}\left(\mathrm{~W} \cdot \mathrm{K}^{-1}\right)$ is the thermal conductance for conductive heat transfer from the laser irradiated part to the nonirradiated part, $K_{r}\left(\mathrm{~W} \cdot \mathrm{K}^{-1}\right)$ is the thermal conductance for radiative heat transfer from the sample surface to the heat reservoir in vacuum, $\varepsilon$ is the hemispherical total emissivity, and $\sigma\left(\mathrm{W} \cdot \mathrm{m}^{-2} \cdot \mathrm{K}^{-4}\right)$ is the Stefan-Boltzmann constant. Subscripts $h$ and $l$, respectively, denote the laser-irradiated part and nonirradiated part. The sample temperature is expressed as the sum of the initial temperature $T_{0}$, the average increase in temperature $\Delta T_{\mathrm{dc}}$ (dc component), and the modulation amplitude $\Delta T_{\mathrm{ac}} \cos (\omega t-\phi)$ (ac component) as

$$
T=T_{0}+\Delta T_{\mathrm{dc}}+\Delta T_{\mathrm{ac}} \cos (\omega t-\phi) .
$$

By solving Eqs. (1) and (2) under the condition of $K_{r} / K_{c} \leq 0.01$, where $K_{r}=A\left(4 \varepsilon \sigma T_{o}^{3}+h\right)$, the temperature amplitude $\Delta T_{\mathrm{ac}}$ and the phase difference $\phi$ between the laser signal and temperature response are expressed as follows ${ }^{7-9)}$ :

$$
\Delta T_{\mathrm{ac}}=\frac{\alpha S_{h} A p_{o}}{\omega C_{p}}\left\{1+\frac{1}{\omega^{2} \tau_{r}^{2}}+\omega^{2} \tau_{c}^{2}\right\}^{-1 / 2}
$$

$$
\cos \phi=\frac{\tau_{c}}{\omega}\left\{\frac{1}{\tau_{c} \tau_{r}}-\omega^{2}\right\}\left\{1+\frac{1}{\omega^{2} \tau_{r}^{2}}+\omega^{2} \tau_{c}^{2}\right\}^{-1 / 2}
$$

Therein, $\tau_{r}(\mathrm{~s})$ is the external thermal relaxation time attributable to the radiative heat transfer and $\tau_{c}(\mathrm{~s})$ is the internal thermal relaxation time attributable to the conductive heat transfer in the droplet. These relaxation times are defined as

$$
\begin{gathered}
\tau_{r}=\frac{C_{p}}{K_{r}}=\frac{C_{p}}{A\left(4 \varepsilon \sigma T_{o}^{3}+h\right)} \\
\tau_{c}=\frac{C_{p}}{K_{c}} V_{h}\left(1-V_{h}\right) \ldots \ldots . . .
\end{gathered}
$$

The term $\alpha S_{h} A p_{\mathrm{o}}$ in Eq. (4) is evaluated quantitatively using the products of the laser power and the normal spectral emissivity at a laser wavelength of the droplet. Assuming Kirchhoff's law, the normal spectral emissivity is used as the absorptivity. For this study, the value of the normal spectral emissivity of liquid stainless steel (SUS 304) is $0.32 \pm 0.005$ at the laser wavelength $(807 \mathrm{~nm})$ measured at $1803 \mathrm{~K}^{11)}$ The distribution of the laser intensity is Gaussian; the $e^{-2}$ radius of the laser beam is $2 \mathrm{~mm}$ for the droplet of $3.5 \mathrm{~mm}$ radius. Effects of the sample curvature on the absorptivity were ignored.

The correction function $f$ is defined as

$$
f=\left\{1+\frac{1}{\omega^{2} \tau_{r}^{2}}+\omega^{2} \tau_{c}^{2}\right\}^{-1 / 2}
$$

The correction function $f$, i.e. the function $\omega \Delta T_{\mathrm{ac}}$, has a maximum value against the modulation frequency as expressed in Eqs. (4) and (8). In general, the condition $\omega^{2} \tau_{r}^{2} \gg 1 \gg \omega^{2} \tau_{c}^{2}$, which satisfies $f \cong 1$, should be achieved by a proper choice of the modulation frequency to determine the heat capacity from Eq. (4). However, in this study, the correction function must be taken into account because of the lower value of $f$. The lower $f$ value is caused by shorter value of $\tau_{r}$; i.e. larger value of $K_{r}$. The forced convection heat transfer of a gas surrounding the droplet is attributable to the larger $K_{r}$ value. The value of $f$ is estimated by using both $\tau_{r}$ and $\tau_{c}$ obtained through the curve fitting of Eq. (5) to the experimental phase difference over the whole frequency range.

\subsection{Thermal Conductivity}

The relation between $\phi$ and $\omega$ as a function of the thermal conductivity, the hemispherical total emissivity of the droplet and the forced convection heat transfer of a gas surrounding the droplet is obtainable by solving the following unsteady heat conduction equation using finite element analysis $^{7-10)}$ :

$$
\begin{aligned}
\rho c_{p} \frac{\partial T}{\partial t}= & \kappa\left[\frac{1}{r^{2}} \frac{\partial}{\partial r}\left(r^{2} \frac{\partial T}{\partial r}\right)\right. \\
& \left.+\frac{1}{r^{2} \sin \theta} \frac{\partial}{\partial \theta}\left(\sin \theta \frac{\partial T}{\partial \theta}\right)\right]+Q(r, \theta)
\end{aligned}
$$


In this equation, $\rho\left(\mathrm{kg} \cdot \mathrm{m}^{-3}\right)$ is the density, $c_{p}\left(\mathrm{~J} \cdot \mathrm{kg}^{-1} \cdot \mathrm{K}^{-1}\right)$ is the isobaric specific heat capacity, $\kappa\left(\mathrm{W} \cdot \mathrm{m}^{-1} \cdot \mathrm{K}^{-1}\right)$ is the thermal conductivity, $Q(r, \theta)\left(\mathrm{W} \cdot \mathrm{m}^{-3}\right)$ is the heat generation rate attributable to electromagnetic induction heating. $r$ (m) and $\theta$ (rad) respectively denote the radial distance and polar angle in the spherical coordinate system.

The following assumptions are made to apply this unsteady heat conduction equation to our experimental condition: (1) the system is axially symmetric; (2) the thermophysical properties are constant within the temperature variation during the calorimetry measurement; (3) laser power is absorbed on the liquid surface depending on its absorptivity; (4) the distribution of laser intensity is Gaussian; (5) the heat transfer in the droplet is governed by conduction.

Boundary conditions (i) of the laser-irradiated area, (ii) of the non-laser-irradiated area, and (iii) of the centerline of the droplet are given as

$$
\text { (i) } \begin{aligned}
-\kappa \frac{\partial T}{\partial n}= & \sigma \varepsilon\left(T^{4}-T_{\infty}^{4}\right)+h\left(T-T_{\infty}\right) \\
& -\frac{2 \alpha P}{\pi r_{\text {laser }}^{2}} \exp \left[-\frac{2 R^{2} \sin ^{2} \theta}{r_{\text {laser }}^{2}}\right]\left(-\mathbf{n} \cdot \mathbf{e}_{\text {laser }}\right)
\end{aligned}
$$

$$
\text { (ii) }-\kappa \frac{\partial T}{\partial n}=\sigma \varepsilon\left(T^{4}-T_{\infty}^{4}\right)+h\left(T-T_{\infty}\right)
$$

and

$$
\text { (iii) }-\kappa \frac{\partial T}{\partial \theta}=0
$$

where $P(\mathrm{~W})$ is the laser power, $R(\mathrm{~m})$ is the radius of the sample, $\mathbf{n}$ is the normal unit vector, and $r_{\text {laser }}(\mathrm{m})$ and $\mathbf{e}_{\text {laser, }}$, respectively, denote the $e^{-2}$ radius and unit vector of the laser. Solving the heat conduction Eq. (9) with the boundary conditions, the phase difference is expressed as

$$
\begin{array}{r}
\phi=\tan ^{-1}\left(\frac{\operatorname{average}\left(\Delta T_{\mathrm{ac}}^{\mathrm{out}}\right)}{\operatorname{average}\left(\Delta T_{\mathrm{ac}}^{\mathrm{in}}\right)}\right) \ldots \ldots \ldots \ldots \ldots . . . . . . . . \\
\operatorname{average}\left(\Delta T_{\mathrm{ac}}^{\mathrm{in}}\right)=\frac{1}{S_{\text {pyrometer }}} \int_{S} \Delta T_{\mathrm{ac}}^{\mathrm{in}}(r, \theta) r \sin \theta d s
\end{array}
$$

and

$$
\operatorname{average}\left(\Delta T_{\mathrm{ac}}^{\mathrm{out}}\right)=\frac{1}{S_{\text {pyrometer }}} \int_{S} \Delta T_{\mathrm{ac}}^{\mathrm{out}}(r, \theta) r \sin \theta d s
$$

where $\Delta T_{\mathrm{ac}}^{\mathrm{in}}$ and $\Delta T_{\mathrm{ac}}^{\mathrm{out}}$, respectively, denote the in-phase and out-of-phase of the temperature amplitude and $S_{\text {pyrometer }}$ is the spot area of the pyrometer.

The variation in $\phi$ with modulation frequency obtained from the above numerical analysis is fitted to the experimental results using a nonlinear least-squares method. In principle, the numerical parameters including the thermal conductivity and emissivity of the droplet can be deter- mined from the fitting process over the entire frequency range. However, in the present study, the heat loss from the droplet surface is not radiation alone; emissivity value is therefore affected by the forced convection heat transfer of the gas. The phase difference at a higher modulation frequency is controlled predominantly by thermal conductivity, although the phase difference at a lower frequency is controlled mainly by both radiation and forced convection heat transfer. Thus, only thermal conductivity was determined in this study.

The heat capacity and density of liquid stainless steel (SUS 304) are required to obtain the thermal conductivity as seen in Eq. (9). The heat capacity determined in the present study and the density measured by Matsumoto et al. ( $\rho$ $\left.\left(\mathrm{kg} \cdot \mathrm{m}^{-3}\right)=-0.616 T+8.11 \times 10^{3}\right)^{12)}$ were used.

\section{Experimental}

Figure 2 presents a schematic illustration of the experimental apparatus. Typical austenitic stainless steel (SUS 304: Japanese Industrial Standards) was used as a sample. The sample was held at the center of an rf coil (max. $15 \mathrm{~kW}$, $200 \mathrm{kHz}$ ), and the coil was equipped at the center of a superconducting magnet with $220 \mathrm{~mm}$ bore (6T220-CSM, SHI, Japan). Chemical compositions of the stainless steel before and after modulation experiment are presented in Table 1. A mixed $\mathrm{Ar}-5 \% \mathrm{H}_{2}$ gas with addition of $\mathrm{He}$ gas was flowed to prevent the oxidation and the overheating of the sample. The initial temperature of the liquid SUS 304 was controlled using the electric power supply to the rf coil and gas flow rate. The flow rates were $2-4 \mathrm{~L} / \mathrm{min}$ for $\mathrm{Ar}-5 \% \mathrm{H}_{2}$ gas and $2-3 \mathrm{~L} / \mathrm{min}$ for $\mathrm{He}$ gas. The heating and levitation of the sample was done using the rf-coil. The superconducting magnet imposed the dc magnetic field of 4-5 T to suppress the convection in the droplet. The top

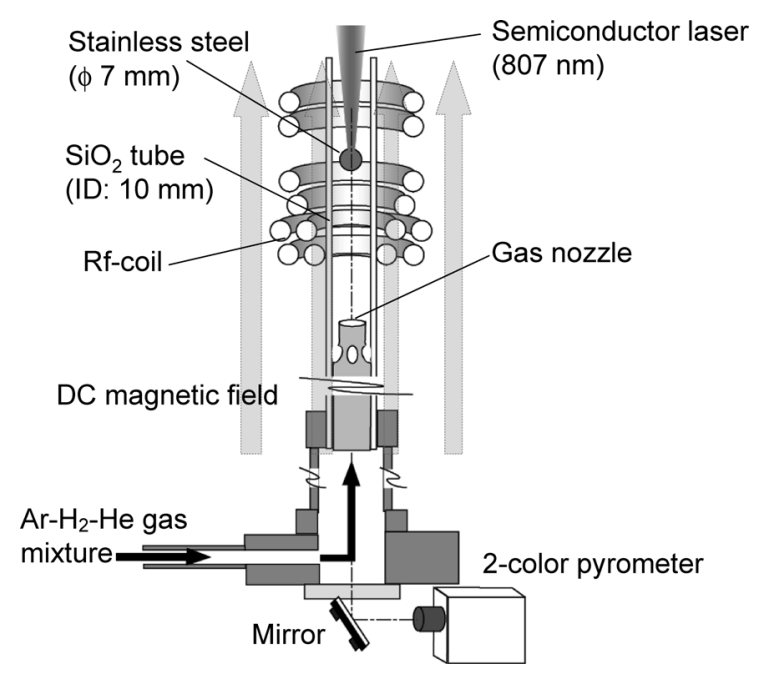

Fig. 2. Schematic illustration for the modulated laser calorimetry of the liquid stainless steel droplet.

Table 1. An example of chemical compositional change of the stainless steel (SUS 304) before and after modulation calorimetry experiment.

(mass \%)

\begin{tabular}{|c|c|c|c|c|c|c|c|c|c|}
\hline & $\mathrm{C}$ & $\mathrm{Si}$ & $\mathrm{Mn}$ & $\mathrm{P}$ & $\mathrm{S}$ & $\mathrm{Ni}$ & $\mathrm{Cr}$ & $\mathrm{Mo}$ & $\mathrm{Cu}$ \\
\hline Before Exp. & 0.04 & 0.31 & 1.31 & 0.029 & 0.006 & 9.92 & 18.1 & 0.18 & 0.32 \\
\hline Affic
\end{tabular}


surface of the levitated liquid SUS 304 was heated sinusoidally using a semiconductor laser through a function generator. The modulation frequency varied from 0.04 to $0.3 \mathrm{~Hz}$. A laser apparatus (wavelength $=807 \pm 3 \mathrm{~nm}$, NBTS140-mk II; JENOPTIK Laser diode Japan Co., Ltd., Tokyo, Japan) was used with a fiber-coupling type cw laser diode. The amplitude of laser power was 31.5-40.1 W. A calibrated laser power meter (Field Mate; Coherent, Inc., Portland, OR, USA) evaluated the net laser power through the optical system, which consisted of an optical fiber and a corrective lens within a standard uncertainty of $1.3 \%$. The temperature response was measured at the bottom of the sample through a gas nozzle with inner diameter of $6 \mathrm{~mm}$ using a two-color pyrometer (IR-CAQ3CS; Chino Corp., Tokyo, Japan) with the emissivity ratio of two wavelengths (1350 and $900 \mathrm{~nm}$ ). The signals from the function generator and the pyrometer were recorded with a sampling interval of $20 \mathrm{~ms}$.

\section{Results}

\subsection{Temperature Response}

Figure 3 shows an example of the temperature response of the noncontact modulated laser calorimetry for the electromagnetically levitated liquid SUS 304. Initially, the droplet temperature was maintained at $T_{0}$ with balancing between the rf induction heating and heat loss caused by radiation and forced convection heat transfer of gas. Then the droplet was heated sinusoidally using the laser. The droplet's temperature gradually increased by $\Delta T_{\mathrm{dc}}$ from $T_{0}$, subsequently, the temperature reached an ac steady state.

An example of the temperature response in the ac steady state is shown in Fig. 4 for the modulation frequency of $0.1 \mathrm{~Hz}\left(0.628 \mathrm{rad} \cdot \mathrm{s}^{-1}\right)$. The temperature amplitude and the phase difference were measured by changing the modulation frequency to obtain both the $\phi-\omega$ and the $\omega \Delta T_{\mathrm{ac}}-\omega$ relation.

Figure 5 shows both the $\phi-\omega$ and the $\omega \Delta T_{\mathrm{ac}}-\omega$ relation obtained from a series of modulation heating. The value of $\omega \Delta T_{\mathrm{ac}}$ has a maximum at a modulation frequency of $0.08 \mathrm{~Hz}$; simultaneously, the phase difference satisfies the requirement that $\phi$ is equal to $-90^{\circ}$ at the frequency, as predicted by the principle of modulation calorimetry. The relaxation times $\tau_{r}$ and $\tau_{c}$ were determined to be $6.6 \mathrm{~s}$ and $0.6 \mathrm{~s}$, respectively by fitting the phase difference to the Eq. (5). Thus, the correction function $f$ was evaluated as a function of frequency using Eq. (8) with two relaxation times, and the maximum value of $f$ was determined to be 0.92 at a frequency of $0.08 \mathrm{~Hz}$. The heat capacity of the liquid SUS 304 was determined from Eq. (4) using the maximum values of $\omega \Delta T_{\mathrm{ac}}$ and $f$.

The experimental frequency dependence of the phase difference designated by an open diamond was well reproduced by the numerical analysis, as designated by a dashed line shown over the whole frequency range. From leastsquares fitting, the thermal conductivity of liquid SUS 304 was determined in the manner described in Sec. 2.2.

\subsection{Specific Heat Capacity}

The results of specific heat capacity and thermal conductivity are summarized in Table 2. From the maximum value

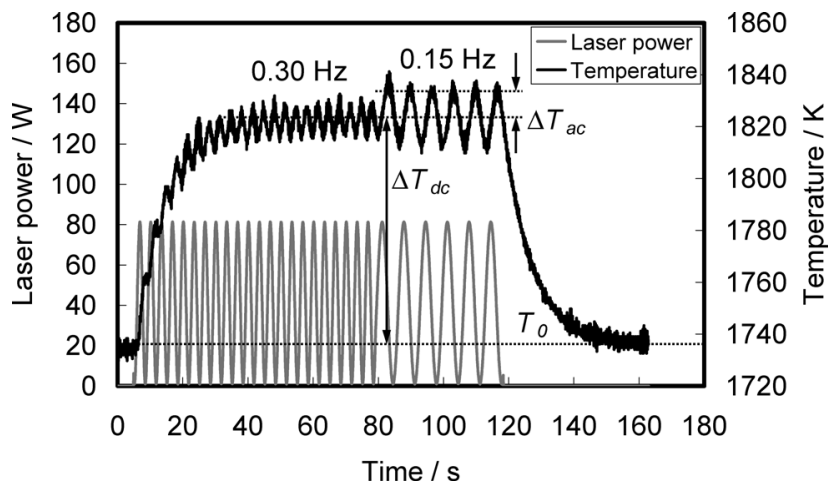

Fig. 3. An example of time dependence of the laser power (lefthand ordinate) and the temperature response (right-hand ordinate) of liquid stainless steel during modulated laser calorimetry.

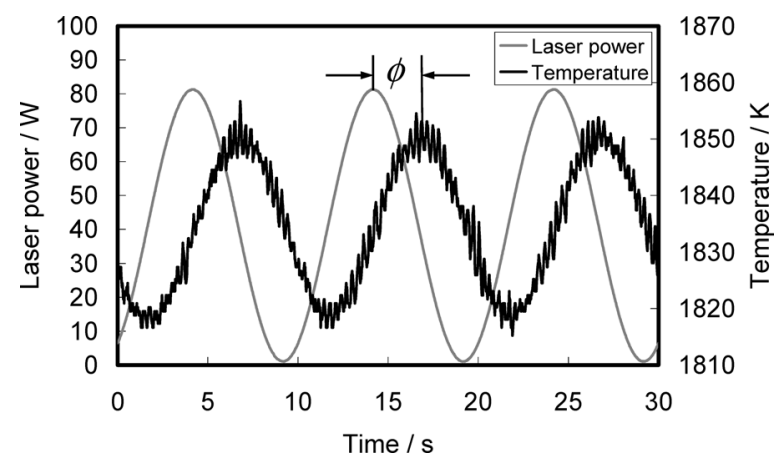

Fig. 4. Temperature response in an ac steady state with modulation frequency of $0.10 \mathrm{~Hz}$.

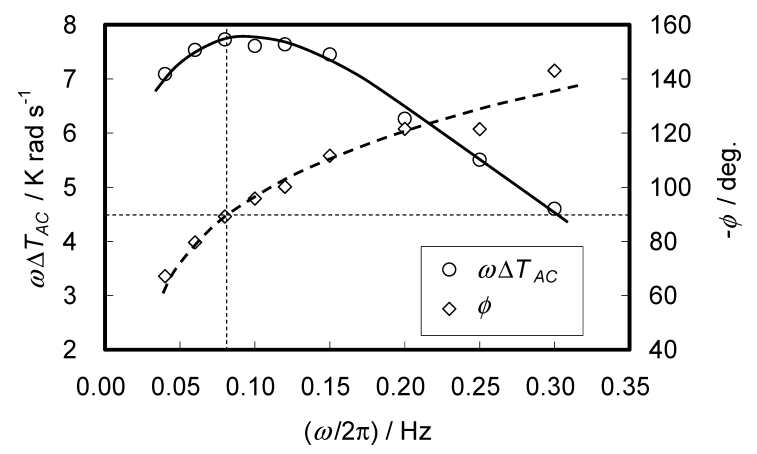

Fig. 5. $\omega \Delta T_{a c}$ as a function of $\omega$ is shown on the left-hand ordinate (open squares). $\phi$ is shown on the right-hand ordinate; experimental data (open diamond), the dashed line shows fitting to the experimental data using the numerical analysis explained in Sec. 2.2.

of $\omega \Delta T_{\mathrm{ac}}$ against the modulation frequency as shown in Fig. 5, the specific heat capacity is determined using Eq. (4) and the value of the absorptivity of liquid SUS 304. Figure 6 shows the temperature dependence of the specific heat capacity of SUS 304. A clear temperature or dc magnetic field dependence of the heat capacity was not observed.

The mean specific heat capacity of liquid SUS 304 is $794 \pm 76 \mathrm{~J} \cdot \mathrm{kg}^{-1} \cdot \mathrm{K}^{-1}$ at temperatures of $1750-1970 \mathrm{~K}$. The experimental uncertainty presented in the above value is double the standard deviation for all the present data. The present results show good agreement with the reference data of $\mathrm{Fe}$ and $\mathrm{Cr}^{13}$ ) within experimental uncertainty. In the solid state, there are two data reported by Cezairliyan and 
Table 2. Experimental conditions and results for the modulation calorimetry.

\begin{tabular}{|c|c|c|c|c|c|c|c|c|c|c|c|c|c|}
\hline \multirow{3}{*}{ Sample No. } & \multirow{2}{*}{$\mathrm{Ar}+\mathrm{H}_{2}$} & \multirow{2}{*}{$\mathrm{He}$} & \multirow{2}{*}{\begin{tabular}{c|} 
rf-coil \\
current
\end{tabular}} & \multirow{2}{*}{$\begin{array}{l}\text { Laser } \\
\text { power }\end{array}$} & \multicolumn{2}{|c|}{ Sample mass } & \multirow{2}{*}{$T_{\text {ave }}$} & \multirow{2}{*}{$\tau_{r}$} & \multirow{2}{*}{$\tau_{c}$} & \multirow{2}{*}{$f_{\max }$} & \multirow{2}{*}{$\alpha$} & \multirow{2}{*}{$c_{p}$} & \multirow{2}{*}{$\kappa$} \\
\hline & & & & & Before exp. & After exp. & & & & & & & \\
\hline & $L \cdot \min ^{-1}$ & $L \cdot \min ^{-1}$ & $A$ & $W$ & $\mathrm{~g}$ & $\mathrm{~g}$ & $\mathrm{~K}$ & $\mathrm{~s}$ & $\mathrm{~s}$ & & & $\mathrm{~J} \cdot \mathrm{kg}^{-1} \cdot \mathrm{K}^{-1}$ & $\mathrm{~W} \cdot \mathrm{m}^{-1} \cdot \mathrm{K}^{-1}$ \\
\hline \multicolumn{14}{|l|}{ Magnetic field $5 \mathrm{~T}$} \\
\hline SUS304-080304b & 2.0 & 2.5 & 641 & 31.5 & 1.475 & 1.431 & 1826 & 6.61 & 0.60 & 0.92 & 0.32 & 826 & 63 \\
\hline SUS304-080304c & 2.0 & 3.0 & 641 & 31.5 & 1.521 & 1.483 & 1801 & 6.34 & 0.63 & 0.91 & 0.32 & 804 & 64 \\
\hline SUS304-080304d & 2.0 & 2.0 & 622 & 31.5 & 1.515 & 1.429 & 1900 & 5.80 & 0.68 & 0.90 & 0.32 & 822 & 55 \\
\hline SUS304-080305b & 2.0 & 2.0 & 680 & 31.5 & 1.538 & 1.483 & 1860 & 6.50 & 0.68 & 0.91 & 0.32 & 810 & 57 \\
\hline SUS304-080305c & 2.0 & 2.5 & 680 & 31.5 & 1.442 & 1.400 & 1782 & 5.90 & 0.66 & 0.90 & 0.32 & 840 & 65 \\
\hline SUS304-080305d & 2.0 & 3.0 & 680 & 31.5 & 1.555 & 1.527 & 1744 & 6.91 & 0.63 & 0.92 & 0.32 & 811 & 62 \\
\hline SUS304-080306a & 2.0 & 2.0 & 641 & 31.5 & 1.504 & 1.424 & 1969 & 6.00 & 0.75 & 0.89 & 0.32 & 754 & 52 \\
\hline SUS304-080306b & 2.0 & 2.0 & 680 & 31.5 & 1.585 & 1.533 & 1824 & 6.80 & 0.70 & 0.91 & 0.32 & 790 & 60 \\
\hline \multicolumn{14}{|l|}{ Magnetic field 4T } \\
\hline SUS304-070320c & 3.9 & 2.0 & 720 & 40.1 & 1.425 & 1.386 & 1892 & 7.30 & 0.48 & 0.94 & 0.32 & 797 & 80 \\
\hline SUS304-070322b & 3.9 & 2.0 & 699 & 40.1 & 1.425 & 1.384 & 1868 & 6.80 & 0.55 & 0.93 & 0.32 & 806 & 74 \\
\hline SUS304-070322c & 2.9 & 2.0 & 699 & 40.1 & 1.425 & 1.388 & 1912 & 6.80 & 0.45 & 0.94 & 0.32 & 786 & 86 \\
\hline SUS304-070322d & 3.9 & 2.0 & 710 & 40.1 & 1.425 & 1.392 & 1847 & 7.00 & 0.60 & 0.92 & 0.32 & 828 & 69 \\
\hline SUS304-070323a & 2.9 & 2.0 & 699 & 40.1 & 1.425 & 1.379 & 1933 & 7.00 & 0.52 & 0.93 & 0.32 & 696 & 78 \\
\hline SUS304-070323b & 3.3 & 2.0 & 699 & 40.1 & 1.425 & 1.389 & 1908 & 7.20 & 0.48 & 0.94 & 0.32 & 751 & 84 \\
\hline
\end{tabular}

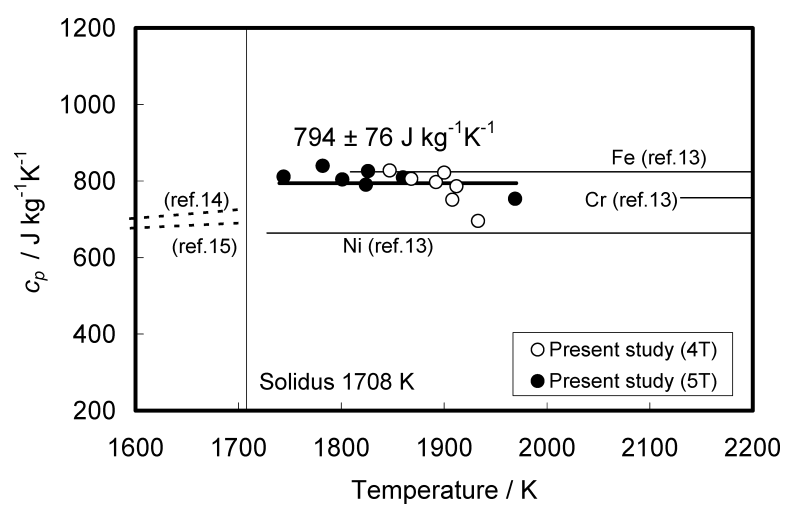

Fig. 6. Isobaric specific heat capacity of the liquid stainless steel (SUS 304) measured in a dc magnetic field at $4 \mathrm{~T}$ (open circles) and $5 \mathrm{~T}$ (solid circles) with data from literature. ${ }^{13-15)}$ The bold line shows the mean value of our experimental data.

Miiller, ${ }^{14)}$ and Zacharia et al., ${ }^{15)}$ however, no experimental data are available in the liquid state except of the present data. The details of the experimental uncertainty will be discussed in Sec. 5.2.

\subsection{Thermal Conductivity}

Figure 7 shows the temperature dependence of the thermal conductivity of liquid SUS 304 together with the previous results reported by other investigators. ${ }^{14-16)}$ The thermal conductivity depends on the dc magnetic field. The thermal conductivity of liquid SUS 304 was decreased with increasing the strength of the static magnetic field. This phenomenon is caused by the suppression of the convection in the droplet by the dc magnetic force. Thus, the data approach the true value with increasing dc magnetic field. However, the present experimental apparatus limits a magnetic field as high as $5 \mathrm{~T}$. In the future work, it is necessary to verify the effect of suppression of the convection by conducting the experiment at higher dc magnetic field and also by the numerical simulation.

The mean thermal conductivity of liquid SUS 304 is $60 \pm 9 \mathrm{~W} \cdot \mathrm{m}^{-1} \cdot \mathrm{K}^{-1}$ under $5 \mathrm{~T}$ at temperatures ranging from 1750 to $1970 \mathrm{~K}$, which is designated by the bold line. The experimental uncertainty presented in the above value is double the standard deviation for all the present data at $5 \mathrm{~T}$.

The thermal conductivities of $\mathrm{Fe}, \mathrm{Ni}$ and $\mathrm{Cr}$ in the liquid state were also presented at their melting points in Fig. 7 for

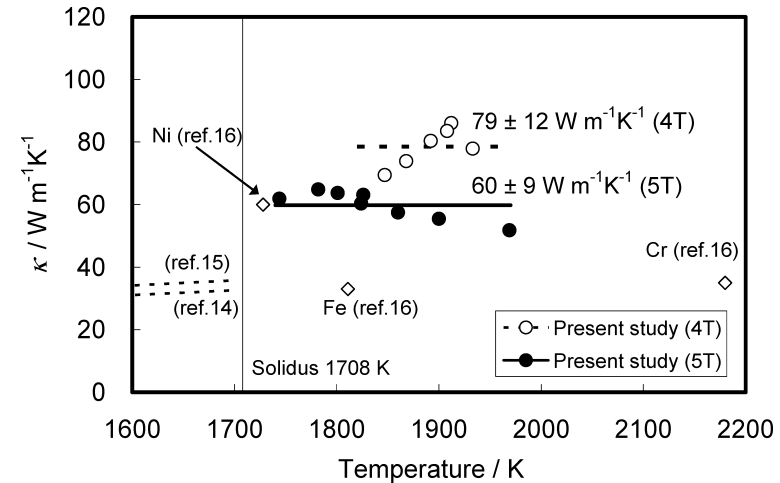

Fig. 7. Thermal conductivity of the liquid stainless steel (SUS 304) measured in a dc magnetic field at $4 \mathrm{~T}$ (open circles) and $5 \mathrm{~T}$ (solid circles) together with data from literature. The bold line shows the mean value of our experimental data obtained at $5 \mathrm{~T}$.

comparison. These are recommended values by Mills et $a l .{ }^{16)}$ The recommended value for liquid $\mathrm{Fe}$ is based on the experimental results obtained using the plane temperature wave technique by Zinovyev et al. ${ }^{16,17)}$ The present data at $5 \mathrm{~T}$ are much higher than that of liquid Fe. No experimental data on liquid SUS 304 are available except of the present data.

The results of the solid SUS 304 were reported by Cezairliyan and Miiller, ${ }^{14)}$ and Zacharia et al. ${ }^{15}$ The data from Cezairliyan and Miiller ${ }^{14)}$ were calculated from the electric conductivity values assuming the WiedemannFranz law.

\section{Discussion}

\subsection{Biot Number and Correction Function}

In the heat flow model explained in the preceding Sec. 2.1 , the forced convection heat transfer coefficient, $h$, is derived from Eq. (6) as,

$$
h=\frac{C_{p}}{4 \pi R^{2} \tau_{r}}-4 \varepsilon \sigma T_{0}^{3}
$$

The value of $h$ was calculated using the above equation with the hemispherical total emissivity value of 0.27 for liquid SUS 304, ${ }^{11}$ and was tabulated in Table 3. The Biot number relevant to the value of $K_{r} / K_{c}$ is defined as follows: 


$$
B i=\frac{4 \varepsilon \sigma T_{0}^{3}+h}{\kappa / R}
$$

The estimated Biot number varies from 0.04 to 0.08 under the present experimental conditions as shown in Table 3. Thus, a quasi-adiabatic condition (i.e. $K_{r} / K_{c} \leq 0.01$ ) is not satisfied well for the modulation calorimetry. Therefore, the correction function $f$ given by Eq. (8) is necessary for determining the heat capacity of the liquid SUS 304 as explained in the Sec. 2.1. The maximum value of $f$ varies from 0.89 to 0.94 depending on the experimental condition as shown in Table 2. All specific heat capacity was corrected using the maximum value of $f$.

\subsection{Uncertainty of Heat Capacity}

The uncertainty in the specific heat capacity was evaluated based on the Guide to the expression of uncertainty in measurement (GUM). ${ }^{18)}$ The specific heat capacity is derived from Eq. (4) as follows:

$$
c_{p}=\frac{\alpha P}{\omega \Delta T_{\mathrm{ac}}} f_{\max } \times \frac{1}{m}
$$

The combined standard uncertainty in the specific heat capacity, $u\left(c_{p}\right)$, is expressed using the following law of propagation of uncertainties:

$$
\begin{gathered}
u^{2}\left(c_{p}\right)=\left(\frac{\partial c_{p}}{\partial \alpha} u(\alpha)\right)^{2}+\left(\frac{\partial c_{p}}{\partial P} u(P)\right)^{2}+\left(\frac{\partial c_{p}}{\partial \omega} u(\omega)\right)^{2} \\
+\left(\frac{\partial c_{p}}{\partial \Delta T_{\mathrm{ac}}} u\left(\Delta T_{\mathrm{ac}}\right)\right)^{2}+\left(\frac{\partial c_{p}}{\partial m} u(m)\right)^{2} \ldots(19)
\end{gathered}
$$

The respective contributions of uncertainty components in the specific heat capacity are listed in Table 4. The sources of uncertainties in $P, \omega$ and $\Delta T_{\text {ac }}$ were based upon the limited resolution of the laser power meter, function generator and pyrometer used in this study. The uncertainty in the sample mass was caused by evaporation during the modulation calorimetry. The uncertainty in the absorptivity was evaluated from the original published data. ${ }^{11)}$ The table shows that uncertainties in $\alpha, \Delta T_{\mathrm{ac}}$ and $m$ are a major contribution. The combined standard uncertainty is evaluated as $34 \mathrm{~J} \cdot \mathrm{kg}^{-1} \cdot \mathrm{K}^{-1}$. The expanded uncertainty at the $95 \%$ level of confidence (coverage factor $=2$ ) in the heat capacity is therefore $68 \mathrm{~J} \cdot \mathrm{kg}^{-1} \cdot \mathrm{K}^{-1}$.

\subsection{Effect of de Magnetic Field on Thermal Conduc- tivity Measurement}

The thermal conductivity of the liquid SUS 304 was measured at dc magnetic fields of $4 \mathrm{~T}$ and $5 \mathrm{~T}$. The thermal conductivity depends on the dc magnetic field, and the lower thermal conductivity was obtained at $5 \mathrm{~T}$ as shown in Fig. 7. This means that the Lorenz force suppressed the convection in the droplet more effectively at $5 \mathrm{~T}$ than at $4 \mathrm{~T}$. The results should be verified experimentally or using numerical simulation.

Tsukada ${ }^{19)}$ demonstrated, using numerical analysis, that the maximum flow rate in the electromagnetically levitated liquid $\mathrm{Si}$ is significantly reduced with increasing strength of the dc magnetic field. In numerical simulation, the electromagnetic field in the system was first computed to obtain the electromagnetic force and the distribution of heat generation rate in the droplet, and then the flow and temperature fields in the droplet were calculated, under the assumptions that the droplet shape is spherical and the system is axisymmetric. Here, the magnetohydrodynamic (MHD) convection was considered as well as the buoyancy and Marangoni convection in the droplet. Moreover, the modulation calorimetry for measuring thermal conductivity was numerically simulated, taking into account the flow field in the droplet. Applying the dc magnetic field of $4 \mathrm{~T}$ is enough to measure the thermal conductivity of liquid Si. However, the magnetic field should be at least $10 \mathrm{~T}$ for measuring the thermal conductivity of liquid Fe. The Prandtle number of liquid $\mathrm{Fe}$ is approximately 10 times larger than that of liquid $\mathrm{Si}$, which contributes the difference. Another possible

Table 3. Evaluation of the forced convection heat transfer coefficient and Biot number for the modulation calorimetry.

\begin{tabular}{|c|c|c|c|c|c|}
\hline \multirow{2}{*}{ Sample No. } & $T_{0}$ & $R$ & $\varepsilon$ & $h$ & $B i$ \\
\cline { 2 - 6 } & $\mathrm{K}$ & $\mathrm{m}$ & & $\mathrm{W} \cdot \mathrm{m}^{-2} \cdot \mathrm{K}^{-1}$ & \\
\hline Magnetic field 5T & & & & & \\
\hline SUS304-080304b & 1726 & 0.0037 & 0.27 & 754 & 0.06 \\
\hline SUS304-080304c & 1701 & 0.0037 & 0.27 & 799 & 0.06 \\
\hline SUS304-080304d & 1800 & 0.0037 & 0.27 & 856 & 0.08 \\
\hline SUS304-080305b & 1760 & 0.0037 & 0.27 & 744 & 0.07 \\
\hline SUS304-080305c & 1682 & 0.0036 & 0.27 & 921 & 0.07 \\
\hline SUS304-080305d & 1644 & 0.0037 & 0.27 & 758 & 0.06 \\
\hline SUS304-080306a & 1869 & 0.0037 & 0.27 & 669 & 0.08 \\
\hline SUS304-080306b & 1724 & 0.0038 & 0.27 & 705 & 0.06 \\
\hline Magnetic field 4T & & & & & \\
\hline SUS304-070320c & 1792 & 0.0036 & 0.27 & 569 & 0.04 \\
\hline SUS304-070322b & 1768 & 0.0036 & 0.27 & 662 & 0.05 \\
\hline SUS304-070322c & 1813 & 0.0036 & 0.27 & 609 & 0.04 \\
\hline SUS304-070322d & 1747 & 0.0036 & 0.27 & 674 & 0.05 \\
\hline SUS304-070323a & 1833 & 0.0036 & 0.27 & 458 & 0.04 \\
\hline SUS304-070323b & 1808 & 0.0036 & 0.27 & 517 & 0.04 \\
\hline
\end{tabular}

Table 4. List of uncertainty components of the specific heat capacity for the experimental condition of SUS304-080304b given in Table 2 .

\begin{tabular}{|c|l|l|c|}
\hline Component & Standard uncertainty & Sensitivity coefficient & $\begin{array}{c}\text { Contribution } \\
\mathrm{J} \cdot \mathrm{kg}^{-1} \cdot \mathrm{K}^{-1}\end{array}$ \\
\hline$\alpha$ & $u(\alpha)=0.005$ & $\partial c_{p} / \partial \alpha=2.58 \times 10^{3} \mathrm{~J} \cdot \mathrm{kg}^{-1} \cdot \mathrm{K}^{-1}$ & 13 \\
\hline$P$ & $u(P)=0.4 \mathrm{~W}$ & $\partial c_{p} / \partial P=26 \mathrm{~s} \cdot \mathrm{kg}^{-1} \cdot \mathrm{K}^{-1}$ & 10 \\
\hline$\omega$ & $u(\omega)=0.00001 \mathrm{rad} \cdot \mathrm{s}^{-1}$ & $\partial c_{p} / \partial \omega=-1.64 \times 10^{3} \mathrm{~J} \cdot \mathrm{kg}^{-1} \cdot \mathrm{K}^{-1} \cdot \mathrm{rad}^{-1} \cdot \mathrm{s}$ & -0.016 \\
\hline$\Delta T_{a c}$ & $u\left(\Delta T_{a c}\right)=0.5 \mathrm{~K}$ & $\partial c_{p} / \partial \Delta T_{a c}=54 \mathrm{~J} \cdot \mathrm{kg}^{-1} \cdot \mathrm{K}^{-2}$ & 27 \\
\hline$m$ & $u(m)=0.022 \times 10^{-3} \mathrm{~kg}$ & $\partial c_{p} / \partial m=-568 \times 10^{3} \mathrm{~J} \cdot \mathrm{kg}^{-2} \cdot \mathrm{K}^{-1}$ & -13 \\
\hline & Combined standard uncertainty & 34 \\
\hline
\end{tabular}


reason is that the larger rf-power was required to levitate liquid Fe than liquid Si. The details of the numerical simulation will be separately reported. Furthermore, the modulation calorimetry will be conducted to measure the thermal conductivity at higher dc magnetic field.

\section{Conclusion}

The newly developed noncontact modulated laser calorimetry method was attempted to measure the specific heat capacity and thermal conductivity of liquid austenitic stainless steel (SUS 304). The specific heat capacity of the liquid SUS 304 was successfully measured as 794士 $76 \mathrm{~J} \cdot \mathrm{kg}^{-1} \cdot \mathrm{K}^{-1}$ at temperatures ranging from 1750 to $1970 \mathrm{~K}$. The uncertainty in the specific heat capacity was evaluated according to the GUM (Guide to the Expression of Uncertainty in Measurement).

The thermal conductivity was measured as $60 \pm 9 \mathrm{~W}$. $\mathrm{m}^{-1} \cdot \mathrm{K}^{-1}$ at $5 \mathrm{~T}$ in the same temperature range. For the thermal conductivity measurement, it is necessary to verify the effect of suppression of the convection by conducting the calorimetry at higher dc magnetic field.

\section{Acknowledgements}

The authors thank Prof. T. Hibiya (Keio University), Prof. M. Watanabe (Gakushuin University), S. Ozawa (Tokyo Metropolitan University), Dr. T. Ohji (Virtual Weld), Dr. T. Baba (AIST), Dr. H. Tanaka (AIST) and Mr. T. Wakamatsu (Daifuku Software Development Co., Ltd) for their helpful comments and discussion. This work is the result of Research and Development to Promote the Creation and Utilization of an Intellectual Infrastructure-Research and Development to Construct the Databases for Computer Aided Welding System, which is supported by the New Energy and Industry Technology Development Organization (NEDO). This development was also supported by ISIJ Research Promotion Grant and SENTAN, JST. This work was performed at the High Field Laboratory for Superconduct- ing Materials, Institute for Materials Research, Tohoku University.

\section{REFERENCES}

1) T. Wakamatsu, S. Ozawa, H. Fukuyama, H. Kobatake, M. Watanabe, A. Mizuno and T. Hibiya: Proc. of the 29th Japan Symposium on Thermophysical Properties, Japan Society of Thermophysical Properties, Tokyo, (2008) 179.

2) H.-J. Fecht and W. L. Johnson: Rev. Sci. Instrum., 62 (1991), 1299.

3) R. K. Wunderlich and H.-J. Fecht: Appl. Phys. Lett., 62 (1993), 3111.

4) R. K. Wunderlich, D. S. Lee, W. K. Johnson and H.-J. Fecht: Phys. Rev. $B, 55$ (1997), 26.

5) R. K. Wunderlich and H.-J. Fecht: Meas. Sci. Technol., 16 (2005), 402.

6) H. Yasuda, I. Ohnaka, Y. Ninomiya, R. Ishii, S. Fujita and K. Kishio: J. Cryst. Growth, 260 (2004), 475.

7) H. Fukuyama, H. Kobatake, K. Takahashi, I. Minato, T. Tsukada and S. Awaji: Meas. Sci. Technol., 18 (2007), 1.

8) H. Kobatake, H. Fukuyama, I. Minato, T. Tsukada and S. Awaji: Appl. Phys. Lett., 90 (2007), 094102-1.

9) H. Kobatake, H. Fukuyama, I. Minato, T. Tsukada and S. Awaji: $J$. Appl. Phys., 104 (2008), 054901-1-8.

10) T. Tsukada, H. Fukuyama and H. Kobatake: Int. J. Heat Mass Transfer, $\mathbf{5 0}$ (2007), 3054.

11) T. Makino, H. Hasegawa, Y. Narumiya, S. Matsuda and T. Kunitomo: Trans. Jpn. Soc. Mech. Eng. (B), 50, (1974), No. 459, 2655.

12) T. Matsumoto, T. Misono, H. Fujii and K. Nogi: J. Mater. Sci., 40 (2005), 2197.

13) M. W. Chase, Jr., ed., NIST-JANAF Thermochemical Tables, 4th ed., The American Chemical Society, Washington, DC and the American Institute of Physics, New York, (1998).

14) A. Cezairliyan and A. P. Miiller: Int. J. Thermophys., 1 (1980), No. 1,83 .

15) T. Zacharia, S. A. David and J. M. Vitek: Metall. Trans. B, 22B (1991), 233.

16) K. C. Mills, B. J. Monaghan and B. J. Keene: Int. Mater. Rev., 41 (1996), No. 6, 209.

17) V. Y. Zinovyev, V. F. Polev, S. G. Taluts, G. P. Zinovyeva and S. A. Ilinykh: Phys. Met. Metallog., 61 (1986), No. 6, 85.

18) L. Kirkup and R. B. Frenkel: An Introduction to Uncertainty in Measurement Using the GUM (Guide to the Expression of Uncertainty in Measurement), Cambridge University Press, Cambridge, (2006).

19) T. Tsukada, K. Sugioka, T. Tsutsumino, H. Fukuyama and H. Kobatake: Int. J. Heat Mass Transfer, 52 (2009), 5152. 